DIGITALCOMMONS - @WAYNESTATE -
Clinical Research in Practice: The Journal of Team Hippocrates

Volume 4 | Issue 1

Article 20

2018

\title{
Intravenous glucocorticoids provide no additional benefit over oral glucocorticoids in chronic obstructive pulmonary disease exacerbation
}

Alexander C. Waselewski

Wayne State University, awaselew@med.wayne.edu

Follow this and additional works at: https://digitalcommons.wayne.edu/crp

Part of the Internal Medicine Commons, Medical Education Commons, Primary Care Commons, and the Translational Medical Research Commons

\section{Recommended Citation}

WASELEWSKI AC. Intravenous glucocorticoids provide no additional benefit over oral glucocorticoids in chronic obstructive pulmonary disease exacerbation. Clin. Res. Prac. 2018 Aug 30;4(1):eP1663. doi: 10.22237/crp/1535500860

This Critical Analysis is brought to you for free and open access by the Open Access Journals at DigitalCommons@WayneState. It has been accepted for inclusion in Clinical Research in Practice: The Journal of Team Hippocrates by an authorized editor of DigitalCommons@WayneState. 


\title{
Intravenous glucocorticoids provide no additional benefit over oral glucocorticoids in chronic obstructive pulmonary disease exacerbation
}

ALEXANDER C. WASELEWSKI, Wayne State University School of Medicine, awaselew@med.wayne.edu

\begin{abstract}
A critical appraisal and clinical application of de Jong YP, Uil SM, Grotjohan HP, Postma DS, Kerstjens HA, van den Berg JW. Oral or IV prednisolone in the treatment of COPD exacerbations: a randomized, controlled, double-blind study. Chest. 2007;132(6):1741-1747. doi: 10.1378/chest.07-0208.
\end{abstract}

Keywords: $\quad$ IV glucocorticoids, oral glucocorticoids, COPD, chronic obstructive pulmonary disease

\section{Clinical Context}

JB is a 68-year-old African American male with significant past medical history of chronic obstructive pulmonary disease (COPD) requiring $4 \mathrm{~L}$ of home oxygen. He has a 30.5 pack year smoking history, type II diabetes mellitus, and hypertension. He presented to the hospital with severe dyspnea and a positive respiratory syncytial virus polymerase chain reaction. He has been hospitalized numerous times in the past for COPD exacerbations most recently in the prior week. His GOLD score was 3 with a forced expiratory volume in the first second $36 \%$ of predicted. His wishes were to be treated to the full extent possible telling the team he wanted to go home. His venous blood gas showed a pCO2 of $76.7 \mathrm{mmHg}$ and a $\mathrm{pH}$ of 6.97 which required admission to the intensive care unit and bi-level positive airway pressure. The patient was started on ipratropium-albuterol nebulizers and completed a 5-day course of 40mg oral prednisone, a standard regimen in COPD exacerbations to prevent readmission and escalation of care. ${ }^{1}$ He showed no improvement and the team decided to try intravenous (IV) methylprednisolone, which did not help either. Intravenous (IV) methylprednisolone was tried because he was clinically deteriorating, and the team wanted to avoid mechanical ventilation.

\section{Clinical Question}

Do IV glucocorticoids have lower rates of treatment failure regarding COPD exacerbations compared to oral glucocorticoids?

\section{Research Article}

de Jong YP, Uil SM, Grotjohan HP, Postma DS, Kerstjens HA, van den Berg JW. Oral or IV prednisolone in the treatment of COPD exacerbations: a randomized, controlled, double-blind study. Chest. 2007;132(6):1741-1747. doi: 10.1378/chest.07-0208

ALEXANDER C. WASELEWSKI is a student in the Wayne State University School of Medicine and a student editor of this journal. 
WASELEWSKI AC. Intravenous glucocorticoids provide no additional benefit over oral glucocorticoids in chronic obstructive pulmonary disease exacerbation. Clin. Res. Prac. 2018 Aug 30;4(1):eP1663. doi: $10.22237 / \mathrm{crp} / 1535500860$
Clinical Research in Practice The Journal of Team Hippocrates

VOL 4 ISS 1 / eP1663 / AUGUST 30, 2018 doi: $10.22237 / \mathrm{crp} / 1535500860$

\section{Related Literature}

To begin the search for articles, PubMed was used with the search phrase, "IV glucocorticoids COPD exacerbation;" 11 relevant articles were selected after reviewing the abstracts. Additionally, UpToDate was searched using "COPD exacerbation treatment," and references browsed for articles not already found through PubMed. PubMed was again searched using the terms, "steroids OR glucocorticoids OR prednisone OR prednisolone AND COPD OR chronic obstructive pulmonary disease AND exacerbation," and was filtered by RCT and Humans. "Best Match" filter was selected and the de Jong article? cited above was again included in the results. When the "Similar Articles" feature was used, no further citations were found.

A total of three relevant studies specifically comparing IV to oral glucocorticoids were found. A prospective, single-blind study conducted by Ceviker and Sayiner compared a 7-day 32mg/day oral methylprednisolone (MP) course with a 4-day course of $1 \mathrm{mg} / \mathrm{kg}$ four times daily IV MP followed by 3 days of $0.5 \mathrm{mg} / \mathrm{kg}$ four times daily IV MP. -3 The authors showed the two courses did not differ in the number of deaths or recurrent exacerbations, but the study was limited with regards to its study population. Only 38 patients were studied, 18 receiving oral therapy and 20 receiving IV, which limits the generalizability of the study and allows for selection bias to distort the outcome because so few of the population of people with COPD were studied. Another limitation is that the two groups did not receive equivalent doses of MP: the oral dosing was considered low dose, while the IV dosing was considered high dose. The oral dose was similar to the treatment protocol defined by GOLD, which is $40 \mathrm{mg}$ prednisone per day for 5 days. 1 The IV dose was considered high dose because it was weight-based and delivered more mg of glucocorticoid per day compared to the fixed oral dose.

In a retrospective cohort study performed by Lindenauer et al. of 79,985 patients with COPD exacerbation, the researchers looked at treatment failure as defined by requiring mechanical ventilation after the second hospital day, death during hospitalization, or a readmission for COPD within 30 days of discharge. 4 Patients received some sort of IV glucocorticoid therapy $(\mathrm{N}=73,765)$, and were compared to 6,220 patients who received oral therapy. Although an enormous number of patients from all over the United States were analyzed in this study, the SORT level of evidence was still only 2 because it was a retrospective study. - One limitation was the lack of standardized treatments for the two groups as ranges of prednisone equivalents were defined for each group rather than a defined dose of a specific glucocorticoid. This means there was variation of dosing between the groups as well as within the groups. Another limitation was that patients were only reviewed for 30 days post discharge rather than 90 days as seen in the other two studies.

de Jong et al conducted the third study, a randomized-controlled trial directly comparing IV and oral glucocorticoids. $\underline{2}$ The article was considered to be the highest quality article found and was chosen for appraisal.

\section{Critical Appraisal}

The study performed by de Jong et al. was a double-blind, double-dummy comparing 103 patients receiving a 5-day course of 60mg oral prednisolone daily to 107 patients receiving a 5-day course of $60 \mathrm{mg}$ IV prednisolone daily. Of the 210 patients analyzed, 29 missed follow-up visits (13 of the IV treatment and 16 of the oral treatment) because of early or late treatment failure, or didn't show up. In the per-protocol analysis, 8 IV patients and 9 oral patients were excluded because they withdrew consent or met one of the exclusion criteria. An intention-to-treat analysis was used to assess the primary outcomes, which mitigated the effect of patients dropping out. After the initial 5-day course, all patients received 30mg prednisolone daily, which was then tapered. During the 5-day course all patients received nebulized ipratropium-albuterol with oral amoxicillin/clavulanate. The double-dummy method was a particular strength of this study because the other studies mentioned had the patients receiving either an IV or oral drug rather than both. An intention-to-treat analysis was used to analyze the data, and a treatment failure percent difference of less than $15 \%$ was used to determine non-inferiority. Based on the description of the study in the methods section it appears that the patients in both were treated as equally as possible. The patient characteristic table showed randomization was successful and our patient was similar to the patients included based on Table 1 of the study.

The inclusion criteria of this study were age $>40$ years, had at least a 10-pack-year history of smoking, and evidence of airflow limitation (FEV1/FVC $<70 \%$ and FEV1 $<80 \%$ predicted). An exacerbation was defined as increased breathlessness with two of the following for $>24$ hours: increased cough frequency or severity, increased sputum volume or purulence, and increased wheeze. Patients were excluded if arterial $\mathrm{pH}<7.26$ or $\mathrm{PaCO} 2>70 \mathrm{mmHg}$, had a history of asthma, participated in previous study within 4 
WASELEWSKI AC. Intravenous glucocorticoids provide no additional benefit over oral glucocorticoids in chronic obstructive pulmonary disease exacerbation. Clin. Res. Prac. 2018 Aug 30;4(1):eP1663. doi: $10.22237 / \mathrm{crp} / 1535500860$
Clinical Research in Practice The Journal of Team Hippocrates

VOL 4 ISS 1 / eP1663 / AUGUST 30, 2018 doi: $10.22237 / \mathrm{crp} / 1535500860$

weeks of admission, already randomized to this study, clinical findings on chest x-ray other than COPD, hypersensitivity to prednisolone, or were totally noncompliant. JB would have been excluded from the study because his $\mathrm{pH}$ was less than 7.26 , but he met all the other criteria. Although his $\mathrm{pH}$ was low, the study is still important to consider in clinical decision making because the patient was stable enough for the general medicine floor. The primary outcome studied was treatment failure, defined as death from any cause, admission to ICU, readmission because of COPD, or intensification of pharmacological treatment. Intensification of pharmacological treatment was use of theophylline, open-label corticosteroids, or antibiotics. Patients were followed for three months after initial hospital treatment. Three months was adequate follow up because the steroid treatment is meant to manage the patient's current exacerbation, not to treat COPD long-term.

Bias was mitigated as much as possible, but one problem for generalizing this study to the clinical scenario above was that the study occurred in the Netherlands. The study also makes no mention of the ethnicities included in the population, which also prohibits the generalizability to this patient's urban population. More time for follow-up would also strengthen the study as COPD is a chronic disease and exacerbations can occur any time over the patient's life. Nonetheless, based on the study design and number of patients in the study a SORT level of evidence of 1 is achieved.

Results of the study showed no significant differences in treatment failure rate as the lower and upper bounds of the $95 \%$ confidence interval included $0 \%$, therefore the criteria for noninferiority were met when comparing oral to IV prednisolone therapy. $61.7 \%$ of IV therapy patients experienced a treatment failure while $56.3 \%$ of oral therapy patients experienced treatment failure. The number of deaths was 5 in the IV group and 2 in the oral group, while hospital readmissions numbered 13 for the IV group and 11 in the oral group. The number needed to treat in this study was 18.5 using oral therapy, but the utility of this number is limited. The study was not designed to see if oral therapy provided better outcomes, but rather to see if oral therapy provided similar outcomes. A number needed to treat would be useful in studies where the authors were trying to prove better outcomes.

\section{Clinical Application}

The de Jong study showed that oral prednisolone was not inferior to IV prednisolone in managing COPD exacerbations with regards to mortality and subsequent hospitalizations. The conclusion of the article is supported by the evidence provided in the results section and is relevant to the case above even though the patient would have been excluded because of the severity of his disease. Once the patient was stable enough for the floor, he expressed wishes to be treated to the full extent possible and had plans to go home. Following the standard of care, he was given oral corticosteroids. The additional course of IV steroids provided no relief. He was given morphine to decrease his dyspnea and was sent to hospice after consultation with his family. The study is relevant to this patient because he received both oral and IV corticosteroids for his COPD exacerbation. Although the study occurred in the Netherlands, the strengths of the study make generalizing the study less hazardous to this patient's clinical scenario.

It could be argued that by treating the patient in the clinical situation above with IV prednisone after oral prednisone a disservice was done to him because redundant therapy was performed based on the conclusion that oral glucocorticoid therapy is equivalent to IV therapy. More money was spent on unnecessary treatment and the patient was exposed to potential infection by giving him IV therapy.

Learning points:

1. Oral glucocorticoid therapy is equivalent to IV therapy in COPD exacerbation.

2. Evidence-based medicine requires use of multiple well-designed studies to support and guide decisionmaking.

3. Managing COPD is difficult and limiting outside disease process such as infection should be considered when deciding therapy. 
WASELEWSKI AC. Intravenous glucocorticoids provide no additional benefit over oral glucocorticoids in chronic obstructive pulmonary disease exacerbation. Clin. Res. Prac. 2018 Aug 30;4(1):eP1663. doi: $10.22237 / \mathrm{crp} / 1535500860$
Clinical Research in Practice The Journal of Team Hippocrates

VOL 4 ISS 1 / eP1663 / AUGUST 30, 2018 doi: $10.22237 / \mathrm{crp} / 1535500860$

\section{References}

1. Global Initiative for Chronic Obstructive Lung Disease. Pocket guide to COPD diagnosis, management, and prevention: a guide for health care professionals (2017 Edition). [uploaded 2016 December, cited 2018 Feb]. https://goldcopd.org/wpcontent/uploads/2016/12/wms-GOLD-2017-Pocket-Guide.pdf. Accessed 2017 Feb 12.

2. de Jong YP, Uil SM, Grotjohan HP, Postma DS, Kerstjens HA, van den Berg JW. Oral or IV prednisolone in the treatment of COPD exacerbations: a randomized, controlled, double-blind study. Chest. 2007;132(6):1741-1747. doi: 10.1378/chest.07-0208

3. Ceviker Y, Sayiner A. Comparison of two systemic steroid regimens for the treatment of COPD exacerbations. Pulmonary Pharmacology \& Therapeutics. 2014;27:179-183. doi: 10.1016/j.pupt.2013.03.004

4. Lindenauer PK, Pekow PS, Lahti MC, Lee Y, Benjamin EM, Rothberg MB. Association of corticosteroid dose and route of administration with risk of treatment failure in acute exacerbation of chronic obstructive pulmonary disease. JAMA. 2010;303(23):2359-2367. doi: 10.1001/jama.2010.796

5. Ebell MH, Siwek J, Weiss BD, Woolf SH, Susman J, Ewigman B, Bowman M. Strength of recommendation taxonomy (SORT): A patient-centered approach to grading evidence in the medical literature. The Journal of the American Board of Family Practice. 2004;17(1):59-67. doi: 10.3122/jabfm.17.1.59 